\title{
Homogeneous Lagrangian submanifolds
}

\author{
Lucio Bedulli And Anna Gori
}

\begin{abstract}
We characterize isometric actions on compact Kähler manifolds admitting a Lagrangian orbit, describing under which condition the Lagrangian orbit is unique. We furthermore give the complete classification of simple groups acting on the complex projective space with a Lagrangian orbit, and we give the explicit list of these orbits.
\end{abstract}

\section{Introduction}

A Lagrangian submanifold of a $2 n$-dimensional symplectic manifold $(M, \omega)$ is an $n$-dimensional submanifold on which the symplectic form $\omega$ vanishes. Lagrangian submanifolds play an important role in symplectic geometry and topology.

In the Kähler setting i.e., when $M$ admits an integrable almost complex structure $J$ such that the bilinear form $g(X, Y)=\omega(X, J Y)$ defines a Riemaniann metric on $M$, the associated Riemannian properties of Lagrangian submanifolds have been studied by different authors (see [7, 16, 25, 26, 31]), in particular in relation to the analysis of minimal Lagrangian submanifolds. In [25] the author asks for a group theoretical machinery producing minimal Lagrangian submanifolds in Hermitian symmetric spaces.

In the present paper we first study the existence problem of homogeneous Lagrangian submanifolds in compact Kähler manifolds, coming to the characterization of isometric actions admitting a Lagrangian orbit, by imposing an additional hypothesis on $M$, holding for a large class of Kähler manifolds including irreducible Hermitian symmetric spaces. Namely we require the space $H^{1,1}(M)$ to be 1-dimensional.

Theorem 1.1. Let $K$ be a compact connected group of isometries acting in a Hamiltonian fashion on a compact Kähler manifold $M$ with $h^{1,1}(M)=1$. Then $M$ admits a $K$-homogeneous Lagrangian submanifold if and only if $K^{\mathbb{C}}$ has an open Stein orbit in $M$.

In $[1$, p. 161$]$ it is proved that if $G:=K^{\mathbb{C}}$ acts holomorphically on a complex manifold with an open Stein orbit, then there exists a totally real 
$K$-orbit $\mathcal{O}$, i.e., at every point of $\mathcal{O}$ the tangent space does not contain complex lines.

A counterexample shows that when $h^{1,1}>1$, even the presence of an open Stein $K^{\mathbb{C}}$-orbit does not guarantee the existence of a Lagrangian $K$-orbit.

When $K$ is semisimple, it turns out that the Lagrangian orbit is unique and, when $M$ is Kähler-Einstein, it is also minimal. In the general case, we describe the actions having infinitely many Lagrangian orbits, characterizing the minimal ones in Section 3.

Theorem 1.2. A Lagrangian $K$-orbit $K \cdot p$ is isolated (actually unique) if and only if the smallest subgroup $K^{\prime}$ of $K$ such that $K \cdot p=K^{\prime} \cdot p$ is semisimple.

Some similar results have been obtained in [27], where it is assumed that there exists a principal Lagrangian orbit.

Our main tool will be the moment map, which can be defined whenever we consider an Hamiltonian group action on $M$. More precisely, let $(M, \omega, J)$ be a compact $2 n$-dimensional Kähler manifold, acted on in a Hamiltonian fashion by a compact connected subgroup $K$ of its full isometry group. This means that there exists a smooth map $\mu: M \rightarrow \mathfrak{k}^{*}=\mathrm{Lie}(\mathrm{K})^{*}$, called a moment map, with the following properties:

1. $d \mu_{p}(v)(X)=\omega_{p}\left(v, \widehat{X}_{p}\right)$ for all $p \in M, v \in T_{p} M$ and $X \in \mathfrak{k}$. Here $\widehat{X}_{p}$ stands for the fundamental field associated to $X$, evaluated at $p$;

2. $\mu$ is $K$-equivariant with respect to the coadjoint action of $K$ on $\mathfrak{k}^{*}$.

If $M$ is connected and $\mu_{1}$ and $\mu_{2}$ are two moment maps, one easily sees that there exists $c$ in the dual of the Lie algebra of the center of $K$, such that $\mu_{1}=\mu_{2}+c$. In general the matter of existence of the moment map is more delicate. However, whenever the Lie group $K$ is semisimple there exists a (unique) moment map (see e.g., [20]). Moreover if $(M, \omega)$, as in our situation, is a compact Kähler manifold and $K$ is a connected compact group of holomorphic isometries then the existence problem can be easily solved: a moment map exists if and only if $K$ acts trivially on the Albanese manifold of $M$ (see e.g., [18]).

In [14] the authors have studied the critical set of the squared moment map $\|\mu\|^{2}$, where $\|\cdot\|$ denotes the norm induced by an $A d(K)$-invariant inner product on $\mathfrak{k}^{*}$. In particular it is proved that if a point $x \in M$ realizes the maximum of $\|\mu\|^{2}$, then the orbit $K \cdot x$ is complex; hence $K \cdot x=K^{\mathbb{C}} \cdot x$ is a closed $K^{\mathbb{C}}$-orbit; it is therefore natural to consider the "dual" problem, i.e., to investigate the $K$-orbits through points $y \in M$ that 
attain the minimum of $\|\mu\|^{2}$. At least when $K$ is semisimple and $K^{\mathbb{C}}$ has an open Stein orbit on $M$, Theorem 1.1 is a step in this direction, since Lagrangian orbits correspond to zeroes of the moment map.

While in Theorem 1.1, we prove the existence of a Lagrangian orbit $L$, we do not exhibit an effective way to single out $L$. At least for self-dual representations, we give in Remark 4.5 an explicit expression, in terms of the highest weight vector, of a point through which the orbit is Lagrangian. Using this result and several ad hoc arguments, we finally give the complete classification of Lagrangian submanifolds of the complex projective space on which a simple group of isometries of the whole space acts transitively.

Theorem 1.3. Let $K$ be a simple compact Lie group acting on the complex projective space $\mathbb{P}(V)$, by means of a unitary representation $\rho: K \rightarrow U(V)$. The group $K$ has a Lagrangian orbit in $\mathbb{P}(V)$ if and only if it appears in Table 1.

The paper is organized as follows. In the second section, we introduce some notations and give the proof of Theorems 1.1 and 1.2. In the third section we analyse the minimality of Lagrangian submanifolds, while in the last section we give the complete classification of simple Lie groups that admit an homogeneous Lagrangian submanifold $L$ in the complex projective space.

Notations and conventions. Lie groups and their Lie algebras will be indicated with capital and gothic letters, respectively. The semisimple part of groups and algebras will be denoted using a subscript $s$. The connected component of the indentity of a Lie group $G$ will be denoted by $G^{o}$. Moreover, after identifying, by means of a $A d(K)$-invariant inner product $\langle$, on $\mathfrak{k}^{*}$, the Lie algebra $\mathfrak{k}$ and its dual $\mathfrak{k}^{*}$, we will alternatively consider $\mu$ as a $\mathfrak{k}$-valued map.

\section{Existence and uniqueness}

Let $M$ be a compact complex manifold with a Kähler form $\omega$ and $K$ be a compact group of isometries acting on $M$ in a Hamiltonian fashion. From now on we fix a moment map $\mu$ and focus on the set of points of $M$ sent by $\mu$ to the center $\mathfrak{z}(\mathfrak{k})$ of $\mathfrak{k}$; we will denote this set by $\mathcal{Z}$. The defining properties of $\mu$ imply that the $K$-orbit $K \cdot p$ is $\omega$-isotropic if and only if $p \in \mathcal{Z}$; indeed, for every $X, Y$ in $\mathfrak{k}$ and $q=k p$ in $K \cdot p$

$$
\omega_{q}\left(\widehat{X}_{q}, \widehat{Y}_{q}\right)=d \mu_{q}\left(\widehat{X}_{q}\right)(Y)=\left.\frac{d}{d t}\right|_{t=0} \exp t X k \cdot \mu(p)(Y) .
$$




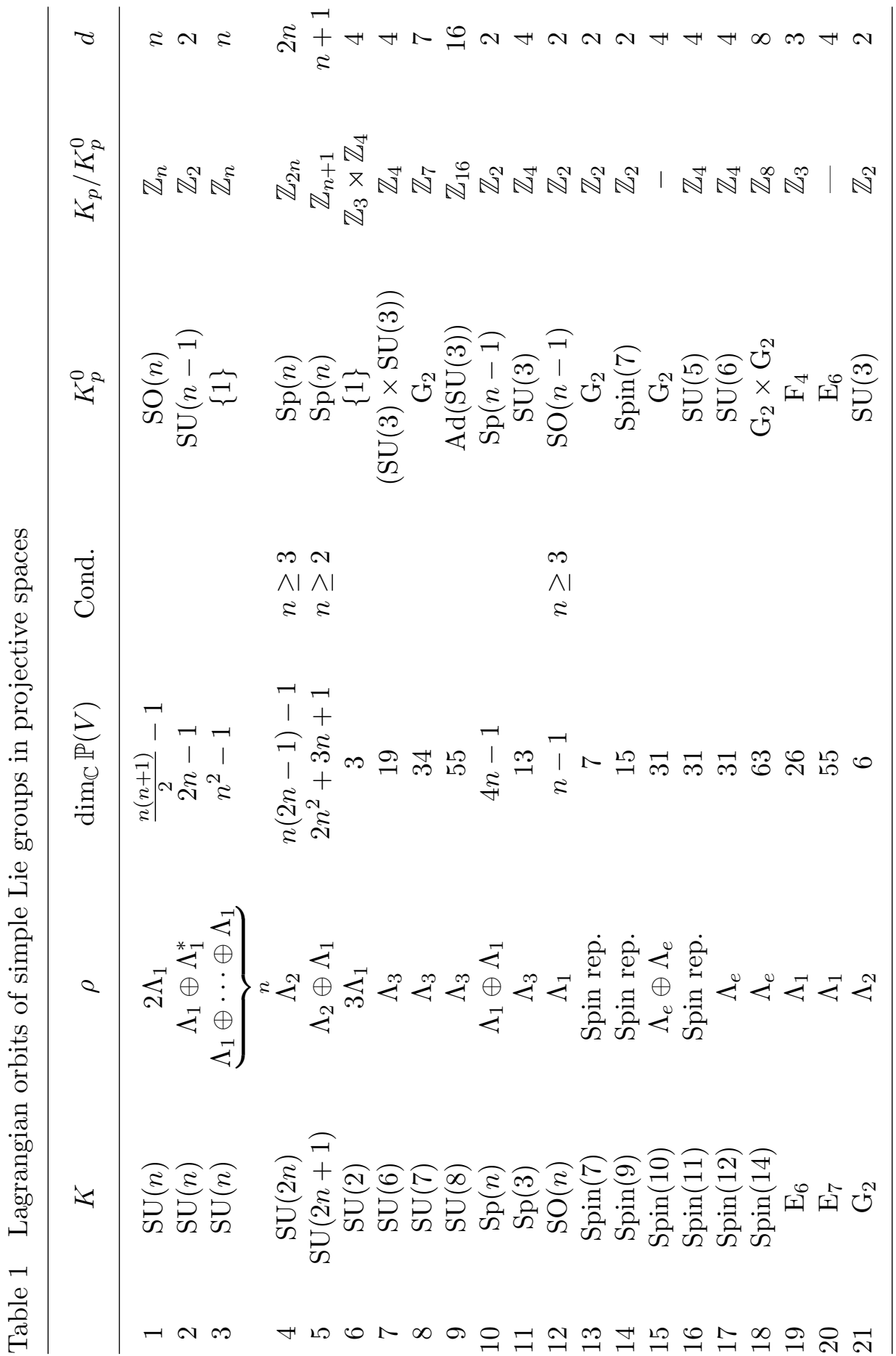


Since the $K$-action on $M$ is holomorphic, it induces, when $M$ is compact, an action of the complexified group $G:=K^{\mathbb{C}}$ on $M$. With these notation we state

Lemma 2.1. Let $p$ be in $\mathcal{Z}$. Then the following statements are equivalent

1. the K-orbit through $p$ is Lagrangian;

2. the $G$-orbit $\Omega$ through $p$ is open in $M$, i.e., $M$ is a $G$-almost homogeneous space.

In this case the G-orbit is a Stein manifold.

Proof. Denote by $\mathcal{O}$ the $K$-orbit through $p$.

$(1) \Rightarrow(2)$ The tangent space to the $G$-orbit through $p$ is given by

$$
T_{p}(G \cdot p)=T_{p} \mathcal{O}+J T_{p} \mathcal{O}
$$

and the sum must be direct since $J T_{p} \mathcal{O} \cap T_{p} \mathcal{O}=\{0\}$ because $\mathcal{O}$ is Lagrangian. Hence $\operatorname{dim} T_{p} G \cdot p=2 \operatorname{dim} T_{p} \mathcal{O}=\operatorname{dim} M$ and the $G$-orbit is open. (2) $\Rightarrow$ (1) Since the $G$-orbit is open, $\operatorname{dim} T_{p} \mathcal{O}+\operatorname{dim} J T_{p} \mathcal{O} \geq 2 n$. Now the conclusion follows recalling that $\mathcal{O}$ is isotropic $(\mu(p) \in \mathfrak{z}(\mathfrak{k}))$, hence $\operatorname{dim} \mathcal{O} \leq n$. Let $H \leq$ $K$ be the isotropy subgroup at $p$. When we consider the complexified action, the Lie algebra of the stabilizer, $\mathfrak{g}_{p}$, is given by the set of vectors $W=$ $X+i Y$ such that $\widehat{W}_{p}=0$. Now, recalling that $J T_{p} \mathcal{O}=\left(T_{p} \mathcal{O}\right)^{\perp}$ we get that $\widehat{X}_{p}=\widehat{Y}_{p}=0$, therefore the complex isotropy is reductive and the open orbit $\Omega=K^{\mathbb{C}} / H^{\mathbb{C}}$ is Stein due to a theorem of Matsushima [24].

An immediate consequence is the following.

Corollary 2.2. The complement of $\Omega$ in $M$ has complex codimension 1 .

Theorem 1.1 proves that, by imposing an additional hypothesis on the cohomology of $M$, the existence of an open Stein $G$-orbit is indeed sufficient to guarantee the presence of a Lagrangian $K$-orbit, while in Theorem 1.2 we characterize the actions having infinitely many Lagrangian orbits. Now we recall two results that will be used in proving the theorems, the first one is due to Kirwan.

Lemma 2.3 [20, p. 97]. Let $x$ and $y$ be two points in a Kähler manifold $M$, acted on in a Hamiltonian fashion by a group of isometries $K$, such that $\mu(x)=\mu(y)=0$. Suppose that $x$ and $y$ lie in different $K$-orbits, then 
there exist two $K^{\mathbb{C}}$-invariant disjoint neighborhoods $U_{x}$ and $U_{y}$ of $x$ and $y$, respectively.

The following is a classical result in Kähler geometry (see e.g., [21, Theorem 7.4] for a proof), it is essentially a consequence of $\partial \bar{\partial}$-lemma, holding for compact Kähler manifolds.

Proposition 2.4. Let $L \rightarrow M$ be a line bundle on a compact Kähler manifold $M$. If $\omega$ is any real, closed $(1,1)$-form such that $[\omega]=c_{1}^{\mathbb{R}}(L) \in H_{d R}^{2}(M)$, then there exists a Hermitian metric along the fibers of $L$ whose curvature form is $\Theta=i /(2 \pi) \omega$.

Now we can prove Theorem 1.1.

Note that a compact Kähler manifold $M$ with $h^{1,1}(M)=1$ is necessarily projective. Indeed, since the Kähler form $\omega$ is of type $(1,1)$, we can scale it so that we obtain an integral class $[\widetilde{\omega}] \in H^{2}(M ; \mathbb{Z})$ and use the Kodaira embedding theorem.

Note that the hypotheses of Theorem 1.1 are naturally satisfied when $M$ is a compact irreducible Hermitian symmetric space.

Proof of Theorem 1.1. We need only to prove that if $G=K^{\mathbb{C}}$ has an open Stein orbit, then there exists a Lagrangian $K$-orbit. Denote again by $\Omega=$ $G \cdot p$ the open Stein orbit and by $Y$ its complement in $M$. By Corollary 2.2, $Y$ is a divisor of $M$ and therefore it determines a holomorphic line bundle $L$ on $M$ and a section $\sigma \in H^{0}(L)$ such that $Y$ is the vanishing locus of $\sigma$. We scale $\omega$ so that we obtain a positive generator of the free part of $H^{2}(M ; \mathbb{Z}) \cap$ $H^{1,1}(M)$. Since $h^{1,1}(M)=1$ the first Chern class of $L$ is a positive integer multiple of the class of the scaled Kähler form $\omega$ on $M$ :

$$
c_{1}(L)=m[\omega] \in H^{1,1}(M) .
$$

Now, by Proposition 2.4, it is possible to find a Hermitian metric $h$ on the fibers of $L$ such that its curvature form is

$$
\Theta=m \frac{i}{2 \pi} \omega .
$$

On the other hand, the curvature on $\Omega$ is exactly (see e.g., [21])

$$
\partial \bar{\partial} \log \|\sigma\|^{2}
$$

where $\|\cdot\|$ is the norm induced by $h$. Thus we have found a strictly plurisubharminic real-valued function $\rho$ such that $\omega=i \partial \bar{\partial} \rho$. Note that by 
construction $\rho: \Omega \rightarrow \mathbb{R}$ is an exhaustion function. Observe that we can assume $\rho$ to be $K$-invariant, since this can be achieved by averaging over the compact group $K$.

Starting from $\rho$ we can define a map $\phi: \Omega \rightarrow \mathfrak{k}^{*}$ as follows

$$
\phi(p)(X):=\frac{1}{2}(J \widehat{X})_{p}(\rho) .
$$

Clearly the $K$-invariance of $\rho$ implies the $K$-equivariance of $\phi$. Moreover, for every $p \in \Omega, v \in T_{p} \Omega$ and $X \in \mathfrak{k}$, we have that $d \phi_{p}(v)(X)=\omega_{p}\left(v, \widehat{X}_{p}\right)$ (see [17] for the proof). Hence, $\phi$ is a moment map for the Hamiltonian action of $K$ on $\Omega$ and therefore its extension to the whole $M$ differs from $\mu$ by an element $z$ of $\mathfrak{z}(\mathfrak{k})$.

Let now $x_{o} \in \Omega$ be a critical point of the exhaustion function $\rho$, then $d \rho_{x_{o}}=0$ and $\phi\left(x_{o}\right)(X)=\frac{1}{2}\left(J \widehat{X}_{x_{o}}\right)(\rho)=0$ for all $X \in \mathfrak{k}$. Thus $\mu\left(x_{o}\right)$ belongs to the Lie algebra of the center of $K$, and the $K$-orbit through $x_{o}$ is Lagrangian by Lemma 2.1 .

Remark 2.5. If the assumption of the Hodge number $h^{1,1}(M)$ in Theorem 1.1 is not satisfied we cannot reach the same conclusion. Indeed consider the example of $\mathrm{SU}(3)$ acting on $\mathbb{P}^{2} \times \mathbb{P}^{2}$ as follows

$$
A \cdot([x],[y])=([A x],[\bar{A} y])
$$

with $A \in \mathrm{SU}(3)$ and $x, y \in \mathbb{C}^{3} \backslash\{0\}$. Since $h^{1,1}\left(\mathbb{P}^{2} \times \mathbb{P}^{2}\right)=2$ we can choose an $\mathrm{SU}(3)$-invariant symplectic form $\omega_{\varepsilon}=\omega_{0} \oplus(1+\varepsilon) \omega_{0}$ on $\mathbb{P}^{2} \times \mathbb{P}^{2}$, where $\omega_{0}$ is the Fubini-Study 2 -form on $\mathbb{P}^{2}$ and $\varepsilon$ is a small positive constant. In this case, there exists an open Stein $G$-orbit (see e.g., [2]), while the image of the moment map does not contain 0 (see also [4] for the picture of the moment polytope in this case).

Remark 2.6. If the group $K^{\mathbb{C}}$ has an open Stein orbit in $M$ with $h^{1,1}(M)=$ 1 , the same is true for $(K \cdot Z)^{\mathbb{C}}$, where $Z$ centralizes $K$. Indeed consider $p \in M$ such that $K \cdot p$ is Lagrangian (cf. Theorem 1.1), then $\mu(p) \in \mathfrak{z}(\mathfrak{k})$ where $\mu$ is a moment map for the $K$-action. On the other hand $\mu$ is the composition of the moment map $\mu^{\prime}$ for the action of $K^{\prime}:=K \cdot Z$ with the projection induced by the inclusion on the dual of the Lie algebras. Therefore $\mu^{\prime}(p) \in \mathfrak{z}\left(\mathfrak{k}^{\prime}\right)$ and for dimensional reasons $K^{\prime} \cdot p$ is Lagrangian and the claim follows from Lemma 2.1.

Assuming the existence of a Lagrangian orbit in the Kähler manifold $M$, we prove Theorem 1.2. 
Proof of Theorem 1.2. In the semisimple case, the moment map is unique, therefore, using the same notation as in Theorem 1.1 we have $\mu\left(x_{0}\right)=0$ at the critical point $x_{o}$ of $\rho$, and $\mu^{-1}(0) \cap \Omega \neq \emptyset$. Take $x$ and $y$ in $\mu^{-1}(0) \cap \Omega$, applying Lemma 2.3, we deduce that $x$ and $y$ belong to the same $K$-orbit, and $\mu^{-1}(0) \cap \Omega$ is therefore compact. Then, since when $M$ is compact the fibers of the moment map are connected [20], we get that $\mu^{-1}(0)$ is contained in $\Omega$ and it is a single $K$-orbit.

If the semisimple part of $K$, which will be denoted by $K_{s}$, has a Lagrangian orbit $L$, then $K$ has a unique Lagrangian orbit. Indeed, combining Theorem 1.1 and the previous remark, we get that there exists a Lagrangian $K$-orbit, this is contained in $\mu_{s}^{-1}(0)=L$, where $\mu_{s}$ is the moment map for the $K_{s}$ action, and it is therefore unique.

Now assume that $K \cdot p$ is a Lagrangian orbit and denote by $H$ the connected component of the identity of the isotropy subgroup $K_{p}$. At the Lie algebra level $\mathfrak{k}$ can be written as the direct sum $\mathfrak{k}_{s} \oplus \mathfrak{z}(\mathfrak{k})$. Consider the projection $\pi: \mathfrak{k} \rightarrow \mathfrak{z}(\mathfrak{k})$. Suppose that $\pi(\mathfrak{h}) \neq 0$, and consider $Z^{\prime} \subset Z$ a subtorus such that its Lie algebra satisfies

$$
\mathfrak{z}(\mathfrak{k})=\pi(\mathfrak{h}) \oplus \mathfrak{z}^{\prime}
$$

and call $K^{\prime}$ the group $K_{s} \cdot Z^{\prime}$. We first prove that $K^{\prime} \cdot p$ has the same dimension of $K \cdot p$ and therefore $K^{\prime} \cdot p$ is Lagrangian. The set of tangent vectors to the $K^{\prime}$-orbit is given by

$$
\widehat{\mathfrak{k}}_{\mid p}=\widehat{\mathfrak{k}}_{s \mid p}+\widehat{\mathfrak{z}}_{\mid p}^{\prime}
$$

while the set of vectors tangent to the $K$-orbit is given by

$$
\widehat{\mathfrak{k}}_{\mid p}=\widehat{\mathfrak{k}}_{s \mid p}+\widehat{\mathfrak{z}}_{\mid p}+\left.\widehat{\pi(\mathfrak{h})}\right|_{\mid p}
$$

By construction $\left.\widehat{\pi(\mathfrak{h})}\right|_{p}$ is contained in $\widehat{\mathfrak{k}}_{s \mid p}$, hence the $K$ - and $K^{\prime}$-orbits through $p$ coincide.

In fact $K^{\prime}$ is the smallest subgroup of $K$ such that $K^{\prime} \cdot p=K \cdot p$. Denote by $H^{\prime}$ the connected component of the identity of $K_{p}^{\prime}$; the projection $\pi\left(\mathfrak{h}^{\prime}\right)$ is $\{0\}$, i.e., $H^{\prime} \subseteq K_{s}$.

Now we assume that $Z^{\prime}$ has dimension at least 1 (i.e., $K^{\prime}$ is not semisimple) and we prove that the Lagrangian orbit $K \cdot p$ is not isolated. Then take a subtorus $Z^{\prime \prime} \leq Z^{\prime}$ of codimension 1 in $Z^{\prime}$ (possibly $Z^{\prime \prime}=\{e\}$ ) and note that for dimensional reasons $K^{\prime \prime}=K_{s} \cdot Z^{\prime \prime}$ has no Lagrangian orbit. Denote 
by $\mu^{\prime \prime}$ the moment map associated to the $K^{\prime \prime}$-action on $M$. Consider the set $M^{H^{\prime}}$, i.e., the set

$$
\left\{x \in M \mid H^{\prime} \cdot x=x\right\} .
$$

We first prove that $\mu^{\prime \prime}\left(M^{H^{\prime}}\right)$ is contained in $\mathfrak{z}_{\mathfrak{m}^{\prime \prime}}\left(\mathfrak{h}^{\prime}\right)$, where $\mathfrak{k}^{\prime \prime}=\mathfrak{h}^{\prime} \oplus \mathfrak{m}^{\prime \prime}$ and $\mathfrak{z}_{\mathfrak{m}^{\prime \prime}}\left(\mathfrak{h}^{\prime}\right)=\left\{X \in \mathfrak{m}^{\prime \prime} \mid\left[X, \mathfrak{h}^{\prime}\right]=0\right\}$. Clearly $\mu^{\prime \prime}\left(M^{H^{\prime}}\right)$ is contained in $\mathfrak{z}_{\mathfrak{k}^{\prime \prime}}\left(\mathfrak{h}^{\prime}\right)$. Moreover let $\gamma(t)$ be a smooth curve contained in $M^{H^{\prime}}$ joining $p$ and a point $x \in M^{H^{\prime}}$. We get

$$
\frac{d}{d t}\left\langle\mu^{\prime \prime}(\gamma(t)), \mathfrak{h}^{\prime}\right\rangle=\left\langle d \mu_{\gamma(t)}^{\prime \prime}\left(\gamma^{\prime}(t)\right), \mathfrak{h}^{\prime}\right\rangle=\omega_{\gamma(t)}\left(\gamma^{\prime}(t), \widehat{\mathfrak{h}}_{\left.\right|_{\gamma(t)}}\right) \equiv 0
$$

where the last equality holds since $\widehat{\mathfrak{h}}_{\mid \gamma(t)}^{\prime}=0$. Now recall that the orbit $K^{\prime} \cdot p$ is Lagrangian hence $\mu^{\prime \prime}(p)=c \in \mathfrak{z}\left(\mathfrak{k}^{\prime \prime}\right)=\mathfrak{z}^{\prime \prime}$ which is orthogonal to $\mathfrak{h}^{\prime}$. Therefore $\mu^{\prime \prime}(x)$ is orthogonal to $\mathfrak{h}^{\prime}$ and belongs to $\mathfrak{m}^{\prime \prime} \cap \mathfrak{z k}_{\mathfrak{k}^{\prime \prime}}\left(\mathfrak{h}^{\prime}\right)=\mathfrak{z}_{\mathfrak{m}^{\prime \prime}}\left(\mathfrak{h}^{\prime}\right)$ as claimed.

Now the dimension of $M^{H^{\prime}}$ is given by

$$
\operatorname{dim} M^{H^{\prime}}=2 \operatorname{dim}\left(K^{\prime} \cdot p\right)^{H^{\prime}}=2\left(\operatorname{dim}\left(K^{\prime \prime} \cdot p\right)^{H^{\prime}}+1\right)=2\left(\operatorname{dim} \mathfrak{z}_{\mathfrak{m}^{\prime \prime}}\left(\mathfrak{h}^{\prime}\right)+1\right) .
$$

This will be used in proving that $Q=\mu^{\prime \prime-1}(c) \cap M^{H^{\prime}}$ is a submanifold.

Note that

$\operatorname{Ker} d \mu_{p}^{\prime \prime}=\left\{Y \in T_{p} M \mid \omega\left(Y, \widehat{X}_{p}\right)=0\right.$ for all $\left.X \in \mathfrak{k}^{\prime \prime}\right\}=\left(J \widehat{\mathfrak{k}}^{\prime \prime}{ }_{p}\right)^{\perp}=T_{p} K^{\prime} \cdot p \oplus V_{1}$,

where $V_{1}$ has dimension 1 and is contained in $T_{p} K^{\prime} \cdot p^{\perp}$, indeed $K^{\prime} \cdot p$ is Lagrangian and $T_{p} M=T_{p} K^{\prime} \cdot p \oplus J \widehat{J k}^{\prime \prime}{ }_{p} \oplus V_{1}$. Moreover $V_{1}$ is contained in $T_{p} M^{H^{\prime}}$; indeed $H^{\prime}$ acts by isotropy on $T_{p} M$ and leaves $\left.\widehat{\mathfrak{k}^{\prime \prime}}\right|_{p}$ invariant hence $\left(\left.J \widehat{\mathfrak{k}}^{\prime \prime}\right|_{p}\right)^{\perp}$ invariant. Therefore $V_{1}$ is $H^{\prime}$-invariant, hence fixed, since it is 1-dimensional and $H^{\prime}$ is compact.

We conclude that

$$
\operatorname{Ker} d \mu_{p}^{\prime \prime} \cap T_{p}\left(M^{H^{\prime}}\right)=\left(T_{p} K^{\prime} \cdot p\right)^{H^{\prime}} \oplus V_{1}
$$

and

$$
\operatorname{dim}\left(\operatorname{Ker} d \mu_{p}^{\prime \prime} \cap T_{p}\left(M^{H^{\prime}}\right)\right)=\operatorname{dim} \mathfrak{z m}_{\mathfrak{m}^{\prime \prime}}\left(\mathfrak{h}^{\prime}\right)+2 .
$$

Counting the dimension of the image, it follows that $\mu^{\prime \prime}{ }_{\mid M^{H^{\prime}}}$ is a submersion at $p$, hence $Q$ is a manifold locally around $p$ whose dimension is $\operatorname{dim} \mathfrak{z m}^{\prime \prime}\left(\mathfrak{h}^{\prime}\right)+$ 2. Note that for dimensional reasons $Q \backslash K^{\prime} \cdot p \neq \emptyset$. To complete the proof it is sufficient to observe that if we take $y \in Q \backslash K^{\prime} \cdot p$, sufficiently close to 
$p$, then the $K^{\prime}$-orbit through $y$ is Lagrangian. Indeed $K^{\prime} \cdot y$ is isotropic for $\mu^{\prime \prime}(y) \in \mathfrak{z}^{\prime \prime}$, and furthermore $H^{\prime} \subseteq K_{y}^{\prime}$; by the Slice Theorem $K_{y}^{\prime o}$ is conjugated to a subgroup of $H^{\prime}$ hence $\operatorname{dim} K_{y}^{\prime} \leq \operatorname{dim} H^{\prime}$ so that $\operatorname{dim} K^{\prime} \cdot y=$ $\operatorname{dim} K^{\prime} \cdot p$.

The uniqueness of the Lagrangian $K$-orbit in the semisimple case is independent of the assumption on $h^{1,1}(M)$.

In fact, using the equivariant version of the Lagrangian embedding theorem (see e.g., [15, p. 315]), we can also state the following proposition holding in the purely symplectic setting.

Proposition 2.7. Let $(M, \omega)$ be a compact connected symplectic manifold acted on in a Hamiltonian fashion by a compact Lie group $K$. Let $\mu: M \rightarrow \mathfrak{k}^{*}$ be a moment map associated to the action. Then, for each $\alpha$ fixed by the coadjoint action of $K$, there exists at most one Lagrangian orbit in $\mu^{-1}(\alpha)$.

Proof. Let $\alpha \in \mathfrak{k}^{*}$ be fixed by $K$. Suppose that $K \cdot x \cong K / H$ be a Lagrangian orbit contained in $\mu^{-1}(\alpha)$. Then, by the equivariant Lagrangian embedding theorem, a neighborhood of $K \cdot x$ in $M$ is equivariantly symplectomorphic to a neighborhood of the zero section in the cotangent bundle $T^{*}(K / H) \simeq$ $K \times_{H}\left(\mathfrak{h}^{0}\right)$, where $\mathfrak{h}^{0}$ denotes the annihilator of $\mathfrak{h}$ in $\mathfrak{k}^{*}$. Up to a constant vector, the moment map $\mu$ on $K \times_{H}\left(\mathfrak{h}^{0}\right)$ is given by $\mu([k, \beta])=k \cdot \beta$. Therefore $\mu^{-1}(0)$ equals the zero section $K \times_{H}\{0\}$. Hence in $M$ the orbit $K \cdot x$ is a connected component of the fiber $\mu^{-1}(\alpha)$. But the level sets of the moment map are connected, hence $K \cdot x=\mu^{-1}(\alpha)$.

A similar argument is also used in the paper [6, Theorem 1.1] now in preparation, where the dimension of the moduli space of Lagrangian orbits is computed.

Coming back to the Kähler case, another remark can be done: whenever the zero level set $\mu^{-1}(0)$ meets the open Stein orbit $\Omega$, we can argue that $\Omega$ coincides with the set of semistable points $M^{\mathrm{ss}}:=\left\{x \in M \mid \overline{G \cdot x} \cap \mu^{-1}(0) \neq\right.$ $\emptyset\}$. Indeed $\Omega$ is always contained in $M^{\text {ss }}$, moreover (see e.g., [30]) $M^{\text {ss }}$ is the smallest $G$-invariant subset of $M$ that contains $\mu^{-1}(0)$, therefore $M^{\text {ss }}=\Omega$. In the Kähler case it is easy to see that the stratum associated to the minimum critical set of $\|\mu\|^{2}$ (see [20, p. 80] for the precise definition) coincides with the set of semistable points. Duistermaat showed (see, for example [22]) that this stratum retracts to the zero set; we have thus proved the following.

Proposition 2.8. If $\mu^{-1}(0) \cap \Omega$ is not empty, then $\Omega$ has $\mu^{-1}(0)$ as a deformation retract, and thus has the same homotopy type of the Lagrangian orbit. 
Remark 2.9. Let $K \cdot p$ be a Lagrangian $K$-orbit. One can easily show that, if $Z \in \mathfrak{z}(\mathfrak{k})$, then $K_{p}=K_{\exp i Z p}$ and all the orbits through $\exp i Z p$ are totally real (i.e., at every point the tangent space is transversal to its image via the complex structure), but not in general Lagrangian. Consider for example the action on $\mathbb{C P}^{N}$, with $N=\left(n^{2}+3 n\right) / 2-1$ induced by the representation $\rho$ of $T^{2} \times S U(n)$ on $V=S^{2}\left(\mathbb{C}^{n}\right) \oplus \mathbb{C}^{n}$, defined by $\rho(g)(X, Y)=\left(\alpha A X A^{t}, \beta A y\right)$ for $g=(\alpha, \beta, A) \in T^{2} \times \mathrm{SU}(n)$, where we see the elements of $S^{2} \mathbb{C}^{n}$ as symmetric matrices. Here there are more than one $K$-Lagrangian orbit but, moving through points $\exp i t Z p$, one does not meet any (other) Lagrangian orbit. Azad, Loeb and Qureshi [3] give necessary and sufficient conditions under which one can prove that there are infinitely many totally real orbits; more precisely this is the case whenever $N_{G}\left(G_{p}\right) / G_{p}$ is not finite. In the non-semisimple case this condition is always satisfied.

Remark 2.10. Whenever the isotropy of a Lagrangian $K$-orbit is discrete, the set of Lagrangian orbits is a manifold whose dimension equals the dimension of the center of the group [27]. This situation holds whenever there exists a regular (i.e., principal or exceptional) Lagrangian $K$-orbit. Nevertheless note that if a Lagrangian orbit $K \cdot p$ is principal and contained in $M_{\mu}$, where $M_{\mu}$ is the set of points $x$ in $M$ whose orbits $K \cdot \mu(x)$ has maximal dimension, then necessarily $K$ must be abelian. Indeed, in general when $p \in M_{\mu} \cap M_{\text {princ }}, K_{\mu(p)} / K_{p}^{o}$ is abelian (see e.g., [18]); since in this case $K \cdot p$ is principal and Lagrangian $K_{p}^{o}$ is trivial, so $K_{\mu(p)}$ is abelian, but $\mu(p) \in \mathfrak{z}(\mathfrak{k})$, hence $K_{\mu(p)}=K$ and the claim follows.

Remark 2.11. As a consequence of Theorem 1.1 we have incidentally proved that linear representations of complex semisimple Lie groups are balanced, in the sense of [32], if and only if they have an open Stein orbit.

\section{Minimality of Lagrangian orbits}

We here give a proof of the minimality of the Lagrangian orbit in the semisimple case, however this can be proved also as a consequence of the more general fact stated in Proposition 3.2.

Proposition 3.1. If $K$ is semisimple and $M$ is Kähler-Einstein, the $K$-orbit is also minimal.

Proof. If $H$ denotes the mean curvature vector of the Lagrangian orbit $\mathcal{O}$, it is known (see Dazord [12]) that the 1-form $\alpha \in \Lambda^{1}(\mathcal{O})$ which is the $\omega$-dual of 
$H$ restricted to $\mathcal{O}$ is closed. But $\alpha$ is $K$-invariant, hence for every $X, Y \in \mathfrak{k}$ we have

$$
0=d \alpha(\widehat{X}, \widehat{Y})=\widehat{X} \alpha(\widehat{Y})-\widehat{Y} \alpha(\widehat{X})-\alpha([\widehat{X}, \widehat{Y}])=-\alpha([\widehat{X}, \widehat{Y}]),
$$

so that $\alpha(\widehat{[\mathfrak{k}, \widehat{\mathfrak{k}}]})=\alpha(\widehat{\mathfrak{k}})=0$ and $\alpha \equiv 0$. This means $H=0$.

Actually one can characterize the minimal Lagrangian orbit $L$ in the general case.

When $(M, \omega)$ is compact we can define a canonical moment map, $\widetilde{\mu}$, that is characterized by the fact that $\int_{M} \mu \omega^{n}=0$. If further $M$ is Kähler-Einstein with Einstein constant $c$, then $\widetilde{\mu}$ can be explicitly written (see e.g., $[13,28]$ ):

$$
\widetilde{\mu}(p)(Y):=\frac{1}{2 c} \operatorname{div}\left(J \widehat{Y}_{p}\right)
$$

for every $Y \in \mathfrak{k}$.

Proposition 3.2. Let $\widetilde{\mu}$ be the canonical moment map of a Kähler-Einstein manifold, then a Lagrangian orbit $\mathcal{O}$ is minimal if and only if $\widetilde{\mu}(\mathcal{O})=0$.

The previous result is stated and proved in [27] assuming that the Lagrangian orbit is principal. Actually Proposition 3.2 holds without any assumption on the type of Lagrangian orbits. Indeed, since $L$ is Lagrangian, in order to prove that $L$ is minimal, it is sufficient to show that the mean curvature vector $H$ at some point $p$ of $L$ is orthogonal to $J \widehat{\mathfrak{k}}_{p}$ as done in [27] in Proposition 3.2. Once an orthonormal frame $\left\{e_{i}\right\}$ at $p$ is fixed, we have

$$
\begin{aligned}
\langle H, J \widehat{Y}\rangle & =\left\langle\nabla_{e_{i}} e_{i}, J \widehat{Y}\right\rangle \\
& =e_{i} \sum\left\langle e_{i}, J \widehat{Y}\right\rangle-\sum\left\langle e_{i}, \nabla_{e_{i}} J \widehat{Y}\right\rangle \\
& =-\sum\left\langle e_{i}, \nabla_{e_{i}} J \widehat{Y}\right\rangle \\
& =-\frac{1}{2} \operatorname{div} J \widehat{Y} \\
& =c \widetilde{\mu}_{p}(Y)=0 .
\end{aligned}
$$

Combining the previous proposition and the fact that the zero level set of the moment map is a single orbit when it meets the open Stein $K^{\mathbb{C}}$-orbit (see proof of Theorem 1.2), we get

Corollary 3.3. Let $K$ be a compact connected group of isometries acting in a Hamiltonian fashion on a compact Kähler-Einstein manifold $M$. Then $M$ admits at most one minimal Lagrangian $K$-orbit. 
Corollary 3.4. Under the same hypotheses of Theorem 1.1, assuming further that $K^{\mathbb{C}}$ is simply connected, $M$ is Kähler-Einstein and the isotropy subgroup $K_{q}^{\mathbb{C}}$ at a point $q$ of the Stein orbit has finite connected components, we get that $M$ admits a unique Lagrangian $K$-orbit which turns out to be minimal.

Proof. From Theorem 1.1 we get that there is a Lagrangian $K$-orbit $L$; moreover any other Lagrangian $K$-orbit has the same homotopy type of $L$ by Proposition 2.8 and therefore has finite fundamental group. But, according to Chen (see [9, Theorem 1.1]), in a Kähler-Einstein manifold, the mean curvature of every compact Lagrangian submanifold with $b_{1}=0$ must vanish somewhere. The homogeneity implies that all the Lagrangian orbits are minimal. The conclusion follows from Corollary 3.3.

Obviously the same result holds if $K^{\mathbb{C}}$ is only supposed to have finite fundamental group.

\section{The classification of simple Lie groups with a Lagrangian orbit in the complex projective space}

In this section we give the complete classification of simple compact Lie groups $K$ with a Lagrangian orbit in the complex projective space. We give also an explicit description of Lagrangian orbits, except in case $K=E_{7}$. This part can be treated combining the results of Section 2 with the work of Sato and Kimura [29] and Kimura [19].

Consider a finite-dimensional unitary representation of a compact Lie group $K$ on a Hermitian vector space $(V,\langle\rangle$,$) . Endow \mathbb{P}(V)$ with the FubiniStudy Kähler form and consider the induced $K$-action. Note that this action is automatically Hamiltonian since $\mathbb{P}(V)$ is simply connected. The map $\mu: \mathbb{P}(V) \rightarrow \mathfrak{k}^{*}$ defined for every $v \in V$ and $X \in \mathfrak{k}$ by

$$
\mu([v])(X)=\frac{1}{i} \frac{\langle X \cdot v, v\rangle}{\langle v, v\rangle}
$$

is a moment map for the $K$-action on $\mathbb{P}(V)$.

Here we recall notations and results from [29]. Given a connected complex linear algebraic group $G$, and a rational representation $\rho$ of $G$ on a finite-dimensional complex vector space $V$, a triplet $(G, \rho, V)$ is prehomogeneous if $V$ has a Zariski dense $G$-orbit. 
We give here an easy-to-prove lemma that allows to find relations between almost homogeneous actions on the projective space and prehomogeneus triplets.

Lemma 4.1. Let $G$ be any complex, connected Lie group. $G$ acts with an open dense orbit on $\mathbb{C P}^{n-1}$ if and only if $G \times \mathrm{GL}(1)$ acts with an open dense orbit on $\mathbb{C}^{n}$, i.e., $\left(G \times \mathrm{GL}(1), \rho, \mathbb{C}^{n}\right)$ is a prehomogeneus triplet.

Hence, due to Theorem 1.1, in order to classify the action of compact simple Lie groups on the projective space admitting a Lagrangian orbit, it is sufficient to go through the list of prehomogeneous triplets in [29], and consider those that have reductive generic isotropy, i.e., those that have an open Stein $G$-orbit. They are exactly regular PV spaces of [29, p. 59]. These spaces are characterized by the existence of a relative invariant, i.e., a rational function $f$ such that there exists a rational character $\chi$ of $G$ satisfying $f(\rho(g) x)=\chi(g) f(x)$ for any $g \in G$ and $x \in V$. We here enclose a lemma that will be useful in the sequel; the proof can be found in [29, p. 64].

Lemma 4.2. If $\rho$ is an irreducible representation, then the polynomial $f$ that defines the hypersurface $Y$ is irreducible.

In [29] prehomogeneous vector spaces are classified up to an equivalence relation which we are going to describe.

Definition 4.3. Two triplets $(G, \rho, V)$ and $\left(G^{\prime}, \rho^{\prime}, V^{\prime}\right)$ are called equivalent if there exist a rational isomorphism $\sigma: \rho(G) \rightarrow \rho^{\prime}\left(G^{\prime}\right)$ and an isomorphism $\tau: V \rightarrow V^{\prime}$, both defined over $\mathbb{C}$ such that the diagram

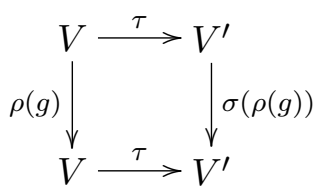

is commutative for all $g \in G$. This equivalence relation will be denoted by $(G, \rho, V) \cong\left(G^{\prime}, \rho^{\prime}, V^{\prime}\right)$.

We say that two triplets $(G, \rho, V)$ and $\left(G^{\prime}, \rho^{\prime}, V^{\prime}\right)$ are castling transforms of each other when there exist a triplet $(\tilde{G}, \tilde{\rho}, V(m))$ and a positive number 
$n$ with $m>n \geq 1$ such that

$$
(G, \rho, V) \cong\left(\tilde{G} \times \mathrm{SL}(n), \tilde{\rho} \otimes \Lambda_{1}, V(m) \otimes V(n)\right)
$$

and

$$
\left(G^{\prime}, \rho^{\prime}, V^{\prime}\right) \cong\left(\tilde{G} \times \operatorname{SL}(m-n), \tilde{\rho}^{*} \otimes \Lambda_{1}, V(m)^{*} \otimes V(m-n)\right),
$$

where $\tilde{\rho}^{*}$ is the dual representation of $\tilde{\rho}$ on the dual vector space $V(m)^{*}$ of $V(m)$. We recall that $V(n)$ is a complex vector space of dimension $n$. A triplet $(G, \rho, V)$ is called reduced if there is no castling transform $\left(G^{\prime}, \rho^{\prime}, V^{\prime}\right)$ with $\operatorname{dim} V^{\prime}<\operatorname{dim} V$.

Note that in fact in each class there is only one representative of the form $G \times \mathrm{GL}(1)$, where $G$ is simple and it is necessarily reduced. This can be seen a posteriori as follows. Suppose that $\left(G^{\prime}, \rho^{\prime}, V^{\prime}\right)$ is a reduced and castling equivalent to $(G \times \mathrm{GL}(1), \rho, V)$, then there should exist a representation $\widetilde{\rho}$ : $\widetilde{G} \rightarrow \mathrm{GL}(V(m))$ such that $(G \times \mathrm{GL}(1), \rho, V) \cong\left(\tilde{G} \times \mathrm{SL}(n), \tilde{\rho} \otimes \Lambda_{1}, V(m) \otimes\right.$ $V(n))$. But now we would have (at least locally) $G \simeq \mathrm{SL}(n)$ and $\mathrm{GL}(1)=\widetilde{G}$ since $G$ is simple, hence $G^{\prime}=\mathrm{GL}(1) \times \mathrm{SL}(m-n)$, but the correspondent triple does not appear in the list of $[29$, p. 144-146].

\subsection{Stabilizer and fundamental group}

We here collect some results and remarks that will be used in order to single out Lagrangian homogeneous submanifolds in the complex projective space.

Assume that a complex Lie group $G=K^{\mathbb{C}}$ acts with an open Stein orbit $\Omega=\mathbb{P}^{n} \backslash Y$ on $\mathbb{P}^{n}$. Denote by $L$ the Lagrangian $K$-orbit. Owing to Proposition 2.8, we get that $\Omega$ has the same homotopy type of $L$. We give here a well-known result on the topology of the complement of an algebraic hypersurface $Y$ in $\mathbb{P}^{n}$ (see e.g., [23]).

Proposition 4.4. Let $Y$ be an algebraic hypersurface of $\mathbb{P}^{n}$. If its irreducible components $Y_{1}, \ldots, Y_{r}$ have degree $d_{1}, \ldots, d_{r}$, respectively, then $H_{1}\left(\mathbb{P}^{n} \backslash\right.$ $Y ; \mathbb{Z})=\mathbb{Z}^{r} /\left(d_{1}, \ldots, d_{r}\right)$.

From the previous proposition it follows that, if $Y$ is irreducible of degree $d>1$, then $H_{1}\left(\mathbb{P}^{n} \backslash Y ; \mathbb{Z}\right)$ is cyclic of order $d$. The open Stein orbit $\mathbb{P}^{n} \backslash Y$ contains the Lagrangian orbit $L=K / K_{p}$ and retracts onto it. From the homotopy sequence, whenever $K$ is simply connected $\pi_{1}\left(K / K_{p}\right) \simeq K_{p} / K_{p}^{o}$. Hence we get a method in order to determine the number of connected 
components of the stabilizer $K_{p}$. If $N_{K}\left(K_{p}^{o}\right) / K_{p}^{o}$ is abelian then $K_{p} / K_{p}^{o}=$ $\mathbb{Z}_{d}$. Indeed $K_{p} / K_{p}^{o} \subset N_{K}\left(K_{p}^{o}\right) / K_{p}^{o}$ is abelian, hence

$$
K_{p} / K_{p}^{o}=\pi_{1}\left(K / K_{p}\right)=H_{1}\left(K / K_{p}\right)=\mathbb{Z}_{d} .
$$

Remark 4.5 (Self-dual representations). Let $V$ be a $(N+1)$-dimensional complex self-dual representation of a compact Lie group $K$ and $\mu$ be the corresponding moment map. Assume that $G=K^{\mathbb{C}}$ has an open Stein orbit $\Omega=G / H$ in $\mathbb{P}^{N}$. Assume also that the highest weight $\lambda$ of the representation satisfies $2 \lambda \notin R^{+}$. Denote by $\mathcal{P}=-\mathcal{P}$ the set of weights. If $v_{ \pm 1} \in V_{ \pm \lambda}$ are two non-zero vectors with the same norm, then $[v]:=\left[v_{1}+v_{-1}\right] \in \mathbb{P}^{N}$ is a point in $\mu^{-1}(0)$ (see [11]). If moreover $2 \lambda \notin R^{+}+R^{+}$, then

$$
\left(\mathfrak{k}_{[v]}\right)^{\mathbb{C}}=\operatorname{Ker} \lambda \bigoplus_{ \pm \alpha \in A_{\lambda}} \mathfrak{k}_{\alpha}
$$

where $A_{\lambda}=\left\{\alpha \in R^{+}:-\lambda+\alpha \notin \mathcal{P}\right\}=\left\{\alpha \in R^{+}:\langle\lambda, \alpha\rangle=0\right\}$. Indeed

$$
X=H+\sum_{\alpha \in \mathbb{R}^{+}} c_{\alpha} E_{\alpha}+\sum_{\alpha \in \mathbb{R}^{-}} d_{\alpha} E_{\alpha}
$$

belongs to $\left(\mathfrak{k}_{[v]}\right)^{\mathbb{C}}$ if and only if

$$
X \cdot v=\lambda(H)\left(v_{1}-v_{-1}\right)+\sum_{\alpha \in R^{+}} c_{\alpha} E_{\alpha} v_{-1}+\sum_{\alpha \in R^{-}} d_{\alpha} E_{\alpha} v_{1}=c \cdot v
$$

and the conclusion follows from the fact that the weight spaces $V_{-\lambda+\alpha}$ and $V_{\lambda-\beta}$ are distinct for $\alpha, \beta \in R^{+}$.

Remark 4.6. If $\Omega=K^{\mathbb{C}} / H^{\mathbb{C}}$ is the open Stein orbit, then there exists $p \in \Omega$ such that $K_{p}=H$. Now, by the $K$-equivariance of $\mu, H=K_{p} \subseteq K_{\mu(p)}$ which is the centralizer of a torus $T$ in $K$.

In some situation the only centralizer of a torus which contains $H$ is the whole group $K$. In this case we have $K_{\mu(p)}=K$ and we can conclude that $\mu(p)=0$ if $K$ is semisimple.

\subsection{The case-by-case classification}

In what follows a compact Lie group $K$ acts on the complex finitedimensional vector space $V$ by a linear representation $\rho$. Moreover we will identify the fundamental highest weights $\Lambda_{l}$ with the corresponding irreducible representations. 
1. $K=\mathrm{SU}(n), \rho=2 \Lambda_{1}$. Identify the representation space $V$ with the set of symmetric $n$ by $n$ complex matrices. Now the Hermitian product on $V$ preserved by $K$ is explicitly given by $\langle A, B\rangle=\operatorname{tr}(A \bar{B})$ and we get immediately $\mu\left(I_{n}\right)=0$. Moreover if $Q$ is the $n$ by $n$ matrix $\operatorname{diag}(-1,1, \ldots, 1)$, the stabilizer at $I_{n}$ is

$$
\left\{\alpha \cdot \operatorname{SO}(n): \alpha^{n}=1\right\} \cup\left\{\alpha Q \cdot \operatorname{SO}(n): \alpha^{n}=-1\right\}
$$

Therefore the $K$-orbit through $I_{n}$ is Lagrangian in $\mathbb{P}(V)$ and $K_{I_{n}} /$ $K_{I_{n}}^{o} \simeq Z_{n}$. Indeed it is generated by $e^{i \pi / n}$ if $n$ is even, and by $e^{i 2 \pi / n}$ if $n$ is odd.

2. $K=\mathrm{SU}(n), \rho=\Lambda_{1} \oplus \Lambda_{1}^{*}$. Identify $V$ with $\mathbb{C}^{n} \oplus \mathbb{C}^{n *}$. Take $p=\left(e_{1}, e_{1}^{*}\right)$. A direct calculation shows that $\mu(p)=0$. The real isotropy is $\mathrm{SU}(n-1) \cdot \mathbb{Z}_{2}$.

3. $K=\mathrm{SU}(n), \rho=\Lambda_{1} \oplus \cdots \oplus \Lambda_{1} n$ times. Identify $V$ with $\mathbb{C}^{n} \oplus \cdots \oplus$ $\mathbb{C}^{n}$. Take $p=\left(e_{1}, e_{2}, \ldots, e_{n}\right)$. A slightly more complicated calculation shows that $\mu(p)=0$. The complex isotropy of $p$ is discrete while the real one is $\mathbb{Z}_{n}$.

4. $K=\mathrm{SU}(2 n), \rho=\Lambda_{2}$. Identify the representation space $V$ with the set of anti-symmetric $2 n$ by $2 n$ complex matrices. The argument of case 4.2 applies to $p=J_{n}=\left[\begin{array}{cc}0 & -I_{n} \\ I_{n} & 0\end{array}\right]$. The real stabilizer is

$$
\left\{\omega \cdot \operatorname{Sp}(n): \omega^{4 n}=1\right\} .
$$

Since $-I_{2 n} \in \operatorname{Sp}(n)$ we have $K_{J_{n}}=\operatorname{Sp}(n) \cdot \mathbb{Z}_{2 n}$ and the $K$-orbit through $J_{n}$ is Lagrangian.

5. $K=\mathrm{SU}(2 n+1), \rho=\Lambda_{2} \oplus \Lambda_{1}$. Identify the $\Lambda_{2}$ part of $V$ with antisymmetric complex matrices and take $p=\left(\widetilde{J}_{n}, e_{1}\right)$ where $\widetilde{J}_{n}=\left[\begin{array}{ll}1 & 0 \\ 0 & J_{n}\end{array}\right]$. Again, if $\mu$ is the moment map associated to the hermitian metric $h((X, v)$,

$(Y, w))=\operatorname{Tr}\left({ }^{t} X \bar{Y}\right)+2^{t} v \bar{w}$, a straightforward computation proves that $\mu(p)=0$, and the real isotropy at $p$ is $\operatorname{Sp}(n) \mathbb{Z}_{n+1}$.

6. $K=\mathrm{SU}(2), \rho=3 \Lambda_{1}$. This case has also been treated in [10] and [5]. The representation is self-dual, hence we apply Remark 4.6. Here $\lambda=3 \epsilon_{1}$ and the set of simple roots $R=\{ \pm \alpha\}$ with $\alpha=\epsilon_{1}-\epsilon_{2}$. Hence $\mathcal{P}$ is the set $\{\lambda, \lambda-\alpha, \lambda-2 \alpha, \lambda-3 \alpha\}$ and $\mathfrak{k}_{[v]}=\{0\}$. Explicitly, identifying the representation space with the space of complex homogeneous polynomial of degree 3 , we can take $[v]=z_{1}^{3}+z_{2}^{3}$ and $K_{[v]}$ is 
a non-abelian group of order 12 whose abelianization is isomorphic to $\mathbb{Z}_{4}$. More precisely $K_{[v]}$ is isomorphic to the unique non-trivial semidirect product $\mathbb{Z}_{3} \rtimes \mathbb{Z}_{4}$ in which $\mathbb{Z}_{3}$ is normal.

7. $K=\mathrm{SU}(6) \rho=\Lambda_{3}$. The representation is again self-dual, here $\lambda=$ $\epsilon_{1}+\epsilon_{2}+\epsilon_{3}$ and $\mathcal{P}=\left\{\epsilon_{i}+\epsilon_{j}+\epsilon_{k} ; i<j<k\right\}$ and $A_{\lambda}=\left\{\epsilon_{i}-\epsilon_{j} ; i<\right.$ $j<3\} \cup\left\{\epsilon_{i}-\epsilon_{j} ; 4 \leq i<j\right\}$ hence $\mathfrak{k}_{[v]}=\mathfrak{s u}(3) \oplus \mathfrak{s u}(3)$. Explicitly $[v]=$ $\left[e_{1} \wedge e_{2} \wedge e_{3}+e_{4} \wedge e_{5} \wedge e_{6}\right]$ and $K_{[v]}$ has four connected components given by

$$
\begin{gathered}
\left\{\left[\begin{array}{cc}
A & 0 \\
0 & D
\end{array}\right]: A, D \in \mathrm{SU}(3)\right\} \cup\left\{\left[\begin{array}{cc}
A & 0 \\
0 & D
\end{array}\right]: A, D \in U(3), \operatorname{det} A=\operatorname{det} D=-1\right\} \\
\cup\left\{\left[\begin{array}{cc}
0 & B \\
C & 0
\end{array}\right]: \operatorname{det} B=\operatorname{det} C=i\right\} \cup\left\{\left[\begin{array}{cc}
0 & B \\
C & 0
\end{array}\right]: \operatorname{det} B=\operatorname{det} C=-i\right\} .
\end{gathered}
$$

Hence the fundamental group of the Lagrangian orbit has order 4 . But, since $H_{1}(L, \mathbb{Z})$ is equal to $\mathbb{Z}_{4}$ (indeed the invariant has degree $4\left[29\right.$, p. 144]), $\pi_{1}(L)=\mathbb{Z}_{4}$.

8. $K=\mathrm{SU}(7), \rho=\Lambda_{3}$. Take $p$ such that $K \cdot p$ is the Lagrangian $K$-orbit in $\mathbb{P}(V)$. By $\left[29\right.$, p. 144] we know that $K_{p}^{o}=\mathrm{G}_{2}$. Let $g \in N_{K}\left(\mathrm{G}_{2}\right)$, then $g$ induces an automorphism of the Lie algebra $\mathfrak{g}_{2}$ which is necessarily inner, since $\mathfrak{g}_{2}$ has only inner automorphisms. Therefore there exists $h \in \mathrm{G}_{2}$ such that $g h$ induces the identity on $\mathfrak{g}_{2}$, i.e., centralizes $\mathrm{G}_{2}$. Now, recalling that $\mathrm{G}_{2}$ acts irreducibly on $\mathbb{C}^{7}$, we get that $g h$ is a scalar multiple of the identity and $N_{K}\left(\mathrm{G}_{2}\right) \subset \mathrm{G}_{2} \cdot \mathbb{Z}_{7}$, where $\mathbb{Z}_{7}$ is the center of $\mathrm{SU}(7)$, and $K_{p}=\mathrm{G}_{2} \cdot \mathbb{Z}_{7}$.

9. $K=\mathrm{SU}(8), \rho=\Lambda_{3}$. In this case, if $p$ is such that $K \cdot p$ is the Lagrangian $K$-orbit in $\mathbb{P}(V)$, following the explicit calculations in [29] (p. 87-90), we know that $K_{p}^{o}$ is the image in $\mathrm{SU}(8)$ of $\mathrm{SU}(3)$ via the $\operatorname{map} A d^{\mathbb{C}}: \operatorname{SU}(3) \rightarrow \operatorname{Aut}(\mathfrak{s l}(3, \mathbb{C}))$, hence $K_{p}^{o} \simeq \mathrm{SU}(3) / \mathbb{Z}_{3}$. We claim that the cardinality of $N_{K}\left(K_{p}^{o}\right) / K_{p}^{o}$ is not greater than 16 , therefore the cardinality of $H_{1}\left(K / K_{p}, \mathbb{Z}\right)$ cannot be greater than 16 , while from [29] we know that its cardinality is exactly 16 . Recall that every automorphism of $\mathfrak{s u}(3)$ is given by the composition of an inner and an outer (the conjugation $\sigma$ ) automorphism; let $g$ be in $N_{K}\left(K_{p}^{o}\right)$ and $\phi_{g}$ the induced automorphism on $K_{p}^{o}$. Then two possibilities arise. In the first case there exists $h \in K_{p}^{o}$ such that $\phi_{g}=\phi_{h}$, in other words $g h^{-1}$ commutes with $K_{p}^{o}$, which acts irreducibly on $\mathbb{C}^{8}$, hence, by the Schur Lemma, it is a scalar multiple of the identity, i.e., an element of the center $\mathbb{Z}_{8}$ of $\mathrm{SU}(8)$. Otherwise there exists 
$h \in K_{p}^{o}$ such that $\phi_{g}=\phi_{h} \circ \sigma$; in this case put $g_{o}=h^{-1} \circ g$. Therefore $N_{K}\left(K_{p}^{o}\right)=K_{p}^{o} \cdot\left(\mathbb{Z}_{8} \cup g_{o} \mathbb{Z}_{8}\right)$, and has at most order 16 . Now, since $K_{p}^{o}$ has no center, we conclude that $K_{p}=K_{p}^{0} \cdot \mathbb{Z}_{16}$.

10. $K=\operatorname{Sp}(n), \rho=\Lambda_{1} \oplus \Lambda_{1}$. Identify $V$ with $\mathbb{C}^{2 n} \oplus \mathbb{C}^{2 n}$. Take $p=\left(e_{1}, e_{2}\right)$, $\mu(p)=0$. The complex isotropy at $p$ is locally isomorphic to $\operatorname{Sp}(n-$ $1, \mathbb{C})$ while the real isotropy is $\operatorname{Sp}(n-1) \cdot \mathbb{Z}_{2}$.

11. $K=\operatorname{Sp}(3), \rho=\Lambda_{3}$. The action is the restriction of the $\mathrm{SU}(6)$ action on the same space. Therefore the stabilizer is given by the intersection of $\mathrm{Sp}(3)$ with the stabilizer obtained in (7). Hence $K_{[v]}$ is

$$
\begin{aligned}
& \left.\left\{\begin{array}{cc}
A & 0 \\
0 & \bar{A}
\end{array}\right]: A \in \mathrm{SU}(3) \operatorname{det} A=1\right\} \\
& \cup\left\{\left[\begin{array}{cc}
A & 0 \\
0 & \bar{A}
\end{array}\right]: A \in U(3), \operatorname{det} A=-1\right\} \\
& \cup\left\{\left[\begin{array}{cc}
0 & B \\
-\bar{B} & 0
\end{array}\right]: B \in U(3) ; \operatorname{det} B=i\right\} \\
& \cup\left\{\left[\begin{array}{cc}
0 & B \\
-\bar{B} & 0
\end{array}\right]: B \in U(3) ; \operatorname{det} B=-i\right\}
\end{aligned}
$$

And we conclude as in (7).

12. $K=\mathrm{SO}(n), \rho=\Lambda_{1}$. The representation $\rho$ is self-dual, nevertheless it is easier to see that $\mu(p)=0$, where $p=[1: 0: \cdots: 0]$. and $K_{p}=$ $\mathrm{SO}(n-1) \cdot \mathbb{Z}_{2}$.

13. $K=\operatorname{Spin}(7), \rho=$ spin rep. The orbits of $\operatorname{Spin}(7)$ are the same of $\mathrm{SO}(8)$ (see the previous case), therefore the Lagrangian orbit is

$$
\frac{\operatorname{Spin}(7)}{\mathrm{G}_{2} \cdot \mathbb{Z}_{2}}=\frac{\mathrm{SO}(8)}{\mathrm{SO}(7) \cdot \mathbb{Z}_{2}}=\mathbb{R P}^{7}
$$

14. $K=\operatorname{Spin}(9), \rho=$ spin rep. The case is completely analogous to the previous one considering the inclusion $\operatorname{Spin}(9) \subset \mathrm{SO}(16)$. Thus the Lagrangian orbit is

$$
\frac{\operatorname{Spin}(9)}{\operatorname{Spin}(7) \cdot \mathbb{Z}_{2}}=\frac{\mathrm{SO}(16)}{\mathrm{SO}(15) \cdot \mathbb{Z}_{2}}=\mathbb{R P}^{15} .
$$

15. $K=\operatorname{Spin}(10), \rho=\Lambda_{e} \oplus \Lambda_{e}$ where $\Lambda_{e}$ is the even half-spin representation. The complex isotropy through the point $p=\left(1+e_{1234}\right.$, $\left.e_{15}+e_{2345}\right)$ is locally isomorphic to $G_{2}^{\mathbb{C}}$ (see [29] also for notations 
and conventions on the spin representation space). Moreover a direct computation using formula (4.1) shows that $\mu(p)=0$, thus $p$ belongs to a Lagrangian orbit.

16. $K=\operatorname{Spin}(11), \rho=$ spin rep. This case and the next one (to which we refer) can be treated simultaneously since $\operatorname{Spin}(11)$ and $\operatorname{Spin}(12)$ have the same orbits on $\mathbb{P}^{31}$. This can be easily seen noting that $\operatorname{Spin}(11) \subset$ Spin(12) and computing the cohomogeneity of these actions. In the case of $\operatorname{Spin}(11)$ the isotropy of the Lagrangian orbit is locally isomorphic to $\mathrm{SU}(5)$.

17. $K=\operatorname{Spin}(12), \rho=\Lambda_{e}$ The computation of the fundamental group of the Lagrangian orbit is done by several steps.

Step 1. The representation $\rho$ is of quaternionic type, so it preserves a quaternionic structure $J \in \operatorname{End}\left(\mathbb{C}^{32}\right)$, such that $J^{2}=-$ id. Denote by $\lambda$ the maximal weight of $\rho$ and by $T$ a fixed maximal torus of $K$. Note first that the Weyl group $W_{\operatorname{Spin}(12)}$ contains -1 . Let $w \in$ $N_{\text {Spin(12) }}(T)$ induce -1 on $\mathfrak{t}$. Since $w(\lambda)=-\lambda, w$ preserves also $\lambda^{\perp}$, therefore $w \in N_{K}(\mathrm{SU}(6)) \subset N_{K}(U(6))$. On the other hand $w$ cannot lie in $U(6)$ because otherwise $w$ should belong to $W_{\mathrm{SU}(6)}$ but $-1 \notin$ $W_{\mathrm{SU}(6)}$. Hence $w$ generates $N(U(6) / U(6)$, and by [8, p. 24] we know that $N_{\mathrm{SO}(12)}(U(6)) / U(6) \simeq \mathbb{Z}_{2}$.

Step 2. Take $p \in \mathbb{P}^{31}$ with $K \cdot p$ Lagrangian and $K_{p}^{o}$ locally isomorphic to $\mathrm{SU}(6)$. Since $\rho$ is self dual, Remark 4.5 implies that $p=u_{1}+$ $u_{-1}$ with $u_{ \pm 1} \in V_{ \pm \lambda}$ and $\left\|u_{1}\right\|=\left\|u_{-1}\right\|$. Now $K_{p} \subset N_{\mathrm{SO}(12)}(\operatorname{SU}(6)) \subset$ $N_{\mathrm{SO}(12)}(U(6))$, hence if $k \in K_{p}$ then $k \in w^{i} U(6)=w^{i} T^{1} \cdot \mathrm{SU}(6)$ for $i=0,1$, where $T^{\prime}$ is the center of $U(6)$.

Step 3. Let $v_{1} \in V_{\lambda}$ be fixed and take $v_{2}=J v_{1} \in V_{-\lambda}$. It is possible to choose $x \in U(1)$ such that $p=v_{1}+x v_{2}$ and $w \cdot p \in \mathbb{C} \cdot p$, and the $K$-orbit through $p$ is Lagrangian.

Step 4. Let $T^{1} \in U(6)$ be the center of $U(6)$. We consider the homomorphism $c: T^{1} \rightarrow U(1)$ such that, for every $t \in T^{1}, t \cdot v_{1}=c(t) \cdot v_{1}$, with $c(t) \neq 1$. By easy computations we get that $t \cdot v_{2}=\overline{c(t)} v_{2}$. Let $k \in K_{p} \subset w^{i} \cdot T^{1} \cdot \mathrm{SU}(6)=T^{1} \cdot w^{i} \mathrm{SU}(6)$, since both $w$ and $\mathrm{SU}(6)$ fix $[p]$, then $k \in H \cdot w^{i} \cdot \mathrm{SU}(6)$ where $H:=\left\{t \in T^{1}, t[p]=[p]\right\}$. Now $t$. $p \in \mathbb{C} \cdot p$ if and only if $c(t)= \pm 1$ i.e., $H=\operatorname{Ker}\left(c^{2}\right) \subset T^{1}$ is cyclic. 
Step 5. Recall that $w^{2} \in T$, hence it commutes with $H$. In $\mathrm{SO}(12), w$ can be taken as $\operatorname{diag}(B, B, \ldots, B)$ where $B=\left[\begin{array}{ll}0 & 1 \\ 1 & 0\end{array}\right]$; then $w \in \operatorname{Spin}(12)$, taken in $\pi^{-1}\left(w_{\mathrm{SO}(12)}\right)$, is such that $w^{2} \in \pi^{-1}(e) \simeq \mathbb{Z}_{2}$ i.e., $w^{4}=i d$.

Step 6. Now we determine $H$. If $u J$, with $u \in i \mathbb{R}$, is a generic element of $\mathfrak{t}^{1}$, and $J:=\operatorname{diag}(A, A, \ldots, A)$ where $A=\left[\begin{array}{cc}0 & 1 \\ -1 & 0\end{array}\right]$ we have $H=\left\{u J \mid \exp (u J) v_{1}= \pm v_{1}\right\}$, and recalling that $\lambda=\frac{1}{2}\left(\omega_{1}+\omega_{2}+\cdots+\right.$ $\left.\omega_{6}\right)$, where $\omega_{i}$ are the fundamental weights, we get

$$
H=\left\{u J \mid u=\frac{\pi}{3} i \cdot k, k \in \mathbb{Z}\right\}
$$

Obviously $H \cap \mathrm{SU}(6)=\mathbb{Z}_{6}$ therefore $[k] \in K_{p} / K_{p}^{o}$ is generated by $[w]$ and by $\alpha$, where $\alpha$ is a non-trivial element of $H / H \cap \mathrm{SU}(6) \simeq \mathbb{Z}_{2}$. Now $w^{2} \in H$, thus $[w]^{2}$ equals $\alpha$ or 1 and $\left|K_{p} / K_{p}^{o}\right| \leq 4$. The claim follows from the fact that the invariant has degree $d=4$.

18. $K=\operatorname{Spin}(14), \rho=\Lambda_{e}$ Let $p$ be such that $K \cdot p$ is the Lagrangian $K$ orbit in $\mathbb{P}(V)$. From $\left[29\right.$, p. 133] we know that $G_{p}^{o}$ is $\mathrm{G}_{2}^{\mathbb{C}} \times \mathrm{G}_{2}^{\mathbb{C}}$ and from the inclusion

$$
\mathrm{G}_{2} \times \mathrm{G}_{2} \subset \mathrm{SO}(7) \times \mathrm{SO}(7) \subset \mathrm{SO}(14)
$$

which lifts to $\operatorname{Spin}(14)\left(\mathrm{G}_{2} \times \mathrm{G}_{2}\right.$ is simply connected), we get $K_{p}^{o}=$ $\mathrm{G}_{2} \times \mathrm{G}_{2}$. Now we claim that $\pi_{1}\left(K_{p}\right)=K_{p} / K_{p}^{o}$ is exactly $\mathbb{Z}_{8}$. Since in this case the degree of the invariant is 8 (see [29, p. 133]) to prove this fact it is sufficient to show that $\left|N_{K}\left(K_{p}^{o}\right) / K_{p}^{o}\right|$ is at most 8 .

First we compute $N_{\mathrm{SO}(14)}\left(K_{p}^{o}\right) / K_{p}^{o}$. As an automorphism of $\mathrm{G}_{2} \times$ $\mathrm{G}_{2}$ an element $g$ of $N_{\mathrm{SO}(14)}\left(K_{p}^{o}\right)$ can either preserve or interchange the $\mathrm{G}_{2}$ factors. Since $\mathfrak{g}_{2}$ has no outer automorphism and the centralizer of $\mathrm{G}_{2}$ in $\mathrm{O}(7)$ is $\{ \pm \mathrm{Id}\}$ we have that $N_{\mathrm{SO}(14)}\left(K_{p}^{o}\right)$ is given by the following four connected components:

$$
\begin{aligned}
& \left\{\left[\begin{array}{cc}
A & 0 \\
0 & B
\end{array}\right]: A, B \in \mathrm{G}_{2}\right\} \cup\left\{\left[\begin{array}{cc}
-A & 0 \\
0 & -B
\end{array}\right]: A, B \in \mathrm{G}_{2}\right\} \\
& \cup\left\{\left[\begin{array}{cc}
0 & A \\
-B & 0
\end{array}\right]: A, B \in \mathrm{G}_{2}\right\} \cup\left\{\left[\begin{array}{cc}
0 & -A \\
B & 0
\end{array}\right]: A, B \in \mathrm{G}_{2}\right\} .
\end{aligned}
$$


Thus $N_{\mathrm{SO}(14)}\left(K_{p}^{o}\right) / K_{p}^{o} \cong \mathbb{Z}_{4}$. Now note that the Lie group covering map $\operatorname{Spin}(14) \rightarrow \mathrm{SO}(14)$ induces an epimorphism

$$
\frac{N_{\mathrm{Spin}(14)}\left(K_{p}^{o}\right)}{K_{p}^{o}} \longrightarrow \frac{N_{\mathrm{SO}(14)}\left(K_{p}^{o}\right)}{K_{p}^{o}}
$$

whose kernel is $\mathbb{Z}_{2}$ and the claim follows.

19. $K=\mathrm{E}_{6}, \rho=\Lambda_{1}$. As before let $p$ be such that $K \cdot p$ is the Lagrangian $K$-orbit in $\mathbb{P}(V)$. From $[29$, p. 138$]$ we know that $G_{p}^{o}$ is $\mathrm{F}_{4}^{\mathbb{C}}$ but $\mathrm{F}_{4} \subset \mathrm{E}_{6}$, hence $K_{p}^{o}=\mathrm{F}_{4}$. Following the same argument as in (8), since $\mathrm{F}_{4}$ has only inner automorphisms, we get that $N_{K}\left(\mathrm{~F}_{4}\right)$ is contained in $\mathrm{F}_{4} \cdot C_{K}\left(\mathrm{~F}_{4}\right)$. Now $\mathrm{F}_{4}$ acts on $\mathbb{C}^{27}=\mathbb{C} \oplus \mathbb{C}^{26}$ irreducibly on the second summand, hence $C_{K}\left(\mathrm{~F}_{4}\right)$ acts on each summand as scalar multiplication. Therefore $C_{K}\left(\mathrm{~F}_{4}\right)$ is contained in a 2-dimensional torus and $N_{K}\left(\mathrm{~F}_{4}\right) / \mathrm{F}_{4}$ is abelian. Since in this case the invariant has degree 3 , we have $K_{p}=\mathrm{F}_{4} \cdot \mathbb{Z}_{3}$. Note that $\mathbb{Z}_{3}$ is the center of $\mathrm{E}_{6}$ which acts trivially on $\mathbb{C P}^{26}$.

20. $K=\mathrm{E}_{7}, \rho=\Lambda_{1}$. As before let $p$ be such that $K \cdot p$ is the Lagrangian $K$-orbit in $\mathbb{P}(V)$. From $[29$, p. 140$]$ we know that $G_{p}^{o}$ is $\mathrm{E}_{6}^{\mathbb{C}}$ but $\mathrm{E}_{6} \subset \mathrm{E}_{7}$, hence $K_{p}^{o}=\mathrm{E}_{6}$. This representation is self-dual.

21. $K=\mathrm{G}_{2}, \rho=\Lambda_{2}$. The orbits of $\mathrm{G}_{2}$ are the same of $\mathrm{SO}(7)$ (see case (4.2)), therefore the Lagrangian orbit is

$$
\frac{\mathrm{G}_{2}}{\mathrm{SU}(3) \cdot \mathbb{Z}_{2}}=\frac{\mathrm{SO}(7)}{\mathrm{SO}(6) \cdot \mathbb{Z}_{2}}=\mathbb{R P}^{6} .
$$

We have thus proved Theorem 1.3.

In the Table 1 , the connected components of the isotropy subgroups $K_{p}$ of points $p$ through which the $K$-orbit is Lagrangian are listed in the fifth column.

\section{Acknowledgment}

The authors would like to thank Prof. F. Podestà for his constant support and useful advices.

\section{References}

[1] D.N. Akhiezer, Lie group actions in complex analysis, Aspects of Mathematics E27, Friedr. Vieweg \& Sohn, Braunschweig, 1995. 
[2] D.N. Akhiezer, Equivariant completions of homogeneous algebraic varieties by homogeneous divisors, Ann. Global Anal. Geom. 2 (1983), 49-78.

[3] H. Azad, J. Loeb and M. Qureshi, Totally real orbits in affine quotients of reductive groups, Nagoya Math. J. 139 (1995), 87-92.

[4] L. Bedulli and A. Gori, On deformations of Hamiltonian actions, Arch. Math. (Basel) 88 (2007), 468-480.

[5] L. Bedulli and A. Gori, A Hamiltonian stable minimal Lagrangian submanifold of projective space with non-parallel second fundamental form, Transform. Groups 12 (2007), 611-617.

[6] L. Biliotti, Homogeneous Lagrangian submanifolds and Hamiltonian actions, Tohoku Math. J. 59 (2007), 603-616.

[7] R. Bryant, Minimal Lagrangian submanifolds of Kähler-Einstein manifolds, Differential geometry and differential equations (Shanghai, 1985), Lecture Notes in Mathematics 1255, Springer, Berlin, 1987, $1-12$.

[8] F. Burstall and J. Rawnsley, Twistor theory for Riemannian symmetric spaces, Lecture Notes in Mathematics 1424, Springer, Berlin, 1990.

[9] B.-Y. Chen, A vanishing theorem for Lagrangian immersions into Einstein-Kaehler manifolds and its applications, Soochow J. Math. 24 (1998), 155-162.

[10] R. Chiang, New Lagrangian Submanifolds of $\mathbb{C P}^{n}$, Int. Math. Res. Not. 45 (2004), 2437-2441.

[11] J. Dadok and V. Kac, Polar representations, J. Algebra 92 (1985), 504-524.

[12] P. Dazord, Sur la géométrie des sous-fibrés et des feuilletages lagrangiens, Ann. Sci. École Norm. Sup. 13 (1981), 465-480.

[13] A. Futaki, Kähler-Einstein metrics and integral invariants, Lecture Notes in Mathematics 1314, Springer, Berlin, 1988.

[14] A. Gori and F. Podestà, A note on the moment map on compact Kähler manifolds, Ann. Global Anal. Geom. 26 (2004), 315-318.

[15] V. Guillemin and S. Sternberg, Symplectic techniques in physics, Cambridge University Press, Cambridge, 1990. 
[16] R. Harvey and H.B. Lawson, Calibrated geometries, Acta Math. 148 (1982), 47-157.

[17] P. Heinzner, A.T. Hukleberry and F. Loose, Kählerian extensions of the symplectic reduction, J. Reine Angew. Math. 455 (1994), 123-140.

[18] A.T. Huckleberry and T. Wurzbacher, Multiplicity-free complex manifolds, Math. Ann. 286 (1990), 261-280.

[19] T. Kimura, A classification of prehomogeneous vector spaces of simple algebraic groups with scalar multiplications, J. Algebra 83 (1983), $72-100$.

[20] F. Kirwan, Cohomology of quotiens in symplectic and algebraic Geometry, Mathematical Notes 31, Princeton University Press, Princeton, 1984.

[21] K. Kodaira and J. Morrow, Complex manifolds, Holt, Rinehart and Winston, Inc., New York, Montreal, London, 1971.

[22] E. Lerman, Gradient flow of the norm squared of a moment map, Enseign. Math. 51 (2005), 117-127.

[23] A. Libgober, Alexander polynomial of plane algebraic curves and cyclic multiple planes, Duke Math. J. 49 (1982), 833-851.

[24] Y. Matsushima, Espaces homogenes de Stein des groupes de Lie complexes, Nagoya Math. J. 16 (1960) 205-218.

[25] Y.-G. Oh, Mean curvature vector and symplectic topology of Lagrangian submanifolds in Einstein-Kähler manifolds, Math. Z. 216 (1994), 471-482.

[26] Y.-G. Oh, Second variation and stabilities of minimal Lagrangian submanifolds in Kähler manifolds, Invent. Math. 101 (1990), 501-519.

[27] T. Pacini, Mean curvature flow, orbits, moment maps, Trans. Amer. Math. Soc. 355 (2003), 3343-3357.

[28] F. Podestà, A note on moment maps and Kähler-Einstein manifolds, Int. J. Geom. Methods Mod. Phys. 3 (2006), 1215-1219.

[29] M. Sato and T. Kimura, A classification of irreducible prehomogeneous vector spaces and their relative invariants, Nagoya Math. J. 65 (1977), $1-155$. 
[30] R. Sjamaar, Holomorphic slices, symplectic reduction and multiplicities of representations, Ann. of Math. 141 (1995), 87-129.

[31] M. Takeuchi, Stability of certain minimal submanifolds of compact Hermitian symmetric spaces, Tohoku Math. J. 36 (1984), 293-314.

[32] N.J. Wildberger, The moment map of a Lie group representation, Trans. Amer. Math. Soc. 330 (1992), 257-268.

Dipartimento di Matematica "U. Dini"

UNIVERSITA' DI FIRENZE"

Viale Morgagni $67 / \mathrm{A}$

50134 FIRENZE

ITALY

E-mail address: bedulli@math.unifi.it

Dipartimento di Matematica "U. Dini"

UNIVERSITA' DI FIRENZE"

Viale Morgagni $67 / \mathrm{A}$

50134 FIRENZE

ITALY

E-mail address: gori@math.unifi.it

ReCeIVED DeCEMBer 1, 2007 
